\title{
Between Dinner and Bedtime
}

National Cancer Institute

\section{Source}

National Cancer Institute. Between Dinner and Bedtime. NCI Thesaurus. Code C92686.

To be done between dinner and the hour of sleep. 